\title{
Abdominopelvic post-irradiation morphea in a prostate cancer patient: the first case of an under-recognized condition
}

\author{
Nicola di Meo ${ }^{1}$, Cecilia Noal ${ }^{1}$, Sara Trevisini ${ }^{1}$, Bruno Ulessi $^{1}$, Giusto Trevisan ${ }^{1}$
}

Received: 30 September 2015 | Returned for modification: 9 October 2015 | Accepted: 23 November 2015

\section{To the Editor:}

Post-irradiation morphea (PIM) is a rare but well-documented under-recognized complication of radiotherapy $(1,2)$. To our knowledge, cases of PIM after radiotheraphy for prostatic carcinoma have never been reported in the literature. A 74-year-old overweight Caucasian male referred to our clinic for sclerotic cutaneous involvement of the abdominopelvic region. He reported a history of radical surgery for prostatic carcinoma (pT2a No Mo) followed by adjuvant radiotherapy (70Gy in 35 fractions) in 2010. One month earlier, an erythematous plaque had developed on the pelvic region and it gradually extended, becoming indurate, thick, and painful. He reported progressive cutaneous incarceration, with motility distress. Upon clinical examination, he presented with an indurate yellowish-white sclerotic plaque involving the upper thighs, pelvic region, and lower abdomen (Figs. 1a, b).

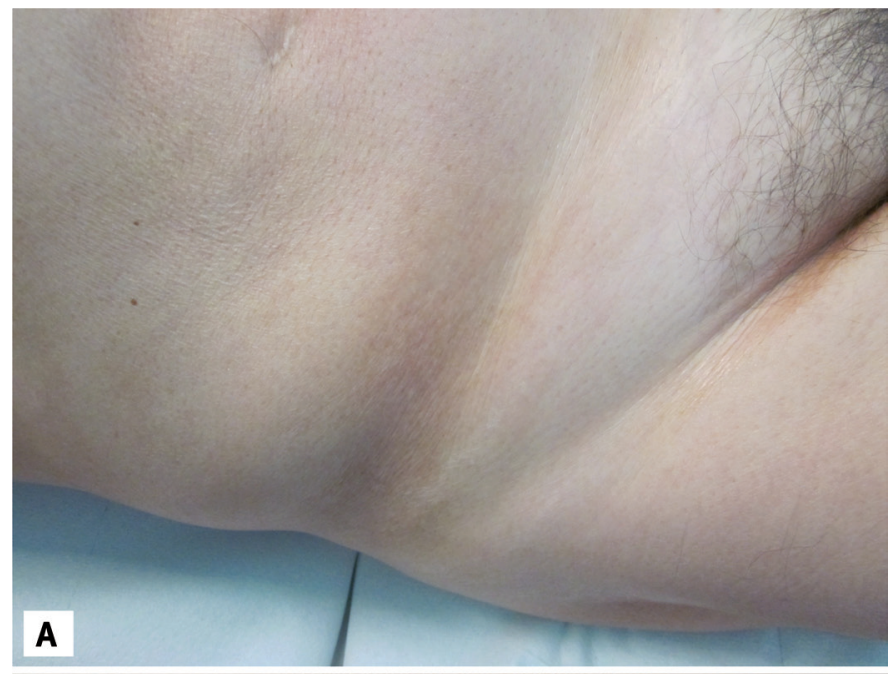

Figure $1 \mathrm{~A}$ and $1 \mathrm{~B} \mid \mathrm{An}$ indurate yellowish-white sclerotic plaque involving the upper thighs, pelvic region, and lower abdomen.
Laboratory investigations were negative for autoantibody profile, hepatitis, and Borrelia burgdorferi. Histological examination revealed a lymphocytic infiltration of the reticular dermis with plasma cells (Fig. 2). Clinical and instrumental exams did not reveal any relapse of cancer or metastatic disease. Because of his anamnesis, clinical findings, and histological features, a diagnosis of PIM was made. The patient started therapy with UVA1 phototherapy associated with daily topical calcipotriol. Clinical improvement was achieved after 3 months, with a mild softening of the skin involved.

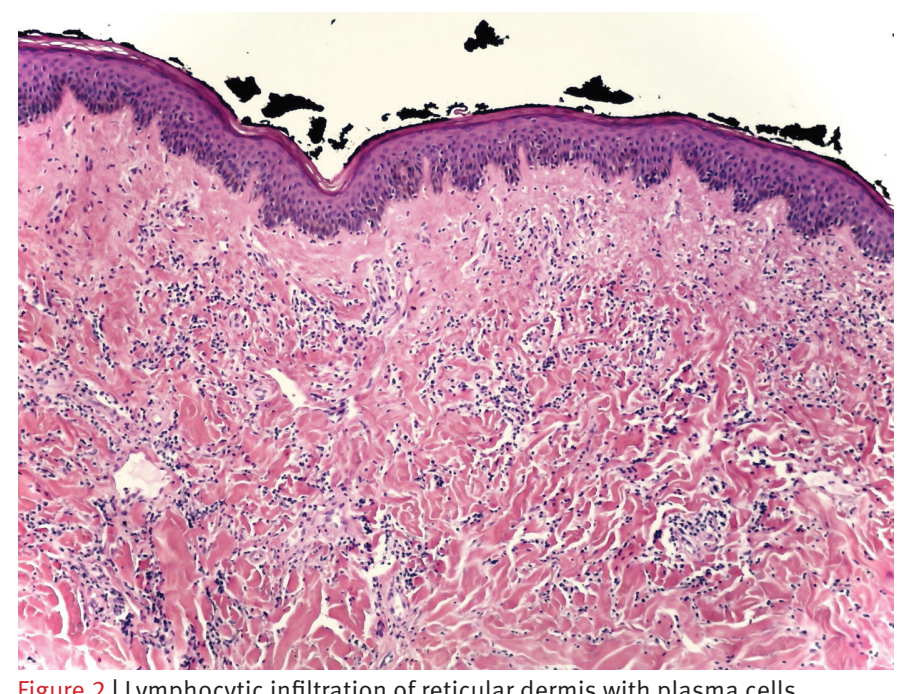

Figure 2 | Lymphocytic infiltration of reticular dermis with plasma cells.

Post-irradiation morphea (PIM) is a rare skin complication of radiotherapy. In a previous study, the incidence of localized morphea following radiotherapy appears to be approximately two out of every 1,00o patients (3). It can occur 1 to 12 months after radiation, and as much as 32 years later. PIM is more frequent in female patients; there has been only one case in a male with subcutaneous lymphoma (4). The majority of PIM occurs in patients treated for breast carcinoma. It is a less common complication after neoadjuvant radiotherapy for head and neck cancer, endometrial cancer, and gastrointestinal neoplasia (5). PIM is characterized by sclerotic plaque, erythema, and induration. Usually it is restricted within the radiation area, but in the literature PIM is reported to extend beyond the irradiated area or involve a distant site (6-7). PIM often presents a difficult diagnosis, especially in non-classical presentations. Radiation-induced fibrosis (RIF), radiation recall dermatitis, post-irradiation sclerodermatous panniculitis, and cancerous sclerosing recurrence are the main differential diagnoses. Skin biopsy results are helpful for distinguishing the disease. PIM occurs later in relation to radiotherapy compared to RIF, which usually appears in the first 3 months. There is usually 
an abrupt onset in PIM, with an initial erythema and thickening that is not observed in RIF (4). Histological findings demonstrated dermal inflammatory infiltrates, which are absent in RIF. Radiation recall dermatitis has been defined as the skin "recalling" previous radiation exposure in response to the administration of drugs (8). The post-irradiation sclerodermatous panniculitis histological pattern reveals significant changes in the subcutaneous tissues with lobular panniculitis. The dermis is nearly unaffected (9).

Treatment of post-irradiation morphea is difficult, and many cases are recalcitrant to therapy. Topical and intralesional corticosteroids, oral and systemic antibiotics, topical hyaluronidase and methotrexate, and chloroquine are used in PIM (10). In some patients, a spontaneous gradual softening of the skin could be observed. In the literature, several studies reported the effectiveness of a combined treatment of calcipotriol and UVA1 irradiation to treat morphea (11). The pathogenesis is still unknown. Davis et al. proposed that radiotherapy induces neoantigen formation, which starts a T-cell response months to years after exposure, stimulating the production of transforming growth factor (TGF-b). TGF-b induces fibroblast activation and the production of extracellular

\section{References}

1. Colver GB, Rodger A, Mortimer PS, Savin JA, Neill SM, Hunter JA. Post-irradiation morphoea. Br J Dermatol. 1989;120:831-5.

2. Verbov J. Post-irradiation morphoea. Br J Dermatol. 1989;121:819-20.

3. Bleasel NR, Stapleton KM, Commens C, Ahern VA. Radiation-induced localized scleroderma in breast cancer patients. Australas J Dermatol. 1999;40:99-102.

4. Schaffer JV, Carroll C, Dvoretsky I, Huether MJ, Girardi M. Postirradiation morphea of the breast presentation of two cases and review of the literature. Dermatology. 2000;200:67-71.

5. Spalek M, Jonska-Gmyrek J, Gatecki J. Radiation-induced morphea-a literature review. J Eur Acad Dermatol Venereol. 2015;29:197-202.

6. Yanaba K, Umezawa Y, Nakagawa H. A case of radiation-induced generalized morphea with prominent mucin deposition and tenderness. Am J Case Rep. 2015;16:279-82.

7. Ardern-Jones MR, Black MM. Widespread morphoea following radiotherapy for carcinoma of the breast. Clin Exp Dermatol. 2003;28:160-2.

8. Hird AE, Wilson J, Symons S, Sinclair E, Davis M, Chow E. Radiation recall dermatitis: case report and review of the literature. Current Oncology. 2008;15:53-62.

9. Carrasco L, Moreno C, Pastor MA, Izquierdo MJ, Fariña C, Martín L, et al. Postirradiation pseudosclerodermatous panniculitis. Am J Dermatopathol. 2001;23: 283-7. matrix proteins, and inhibits the degradation of matrix proteins, implying extensive fibrosis (12). Hermann et al. suggest that there is a higher secretion of Th2cytokines (interleukin 4, interleukin 5), which stimulate collagen synthesis (13). Another theory explains the development of PIM due to a premature terminal differentiation of fibroblast to myofibroblast (14). There seem to be no clear predictive factors predicting the development of RIM. Radiotherapy total dose, age of the patient, dose per fraction, acute skin side effects, or history of autoimmune diseases appear not to be important risk factors (10). Breast size seems to play a role in the development of PIM, perhaps because large breasts have a higher fat content or the radiotherapy dose is inhomogeneous (15). Similar anatomical conditions can be detected in an overweight patient, as in our case. PIM is a rare complication with important adverse side effects of radiotherapy. In the literature, PIM has mainly been described in females treated for breast cancer and it extends within the irradiated area. Considering the amount of radiotherapy for prostate cancer since its introduction, it is possible that our case is the first case of an under-recognized condition.

10. Alhathlool A, Hein R, Andres C, Ring J, Eberlein B. Post-irradiation morphea: case report and review of the literature. J Dermatol Case Rep. 2012;6:73-7.

11. Tay YK. Topical calcipotriol ointment in the treatment of morphea. J Dermatolog Treat. 2003;14:219-21.

12. Davis DA, Cohen PR, McNeese MD, Duvic M. Localized scleroderma in breast cancer patients treated with supervoltage external beam radiation: radiation port scleroderma. J Am Acad Dermatol. 1996;35:923-7.

13. Herrmann T, Gunther C, Csere P. Localized morphea-a rare but significant secondary complication following breast cancer radiotherapy. Case report and review of the literature on radiation reaction among patients with scleroderma/ morphea. Strahlenther Onkol. 2009;185:603-7.

14. Reddy SM, Pui JC, Gold LI, Mitnick HJ. Postirradiation morphea and subcutaneous polyarteritis nodosa: case report and literature review. Semin Arthritis Rheum. 2005;34:728-34.

15. Clarke D, Martinez A, Cox RS. Analysis of cosmetic results and complications in patients with stage I and II breast cancer treated by biopsy and irradiation. Int J Radiat Oncol Biol Phys. 1983;9:1807-13. 